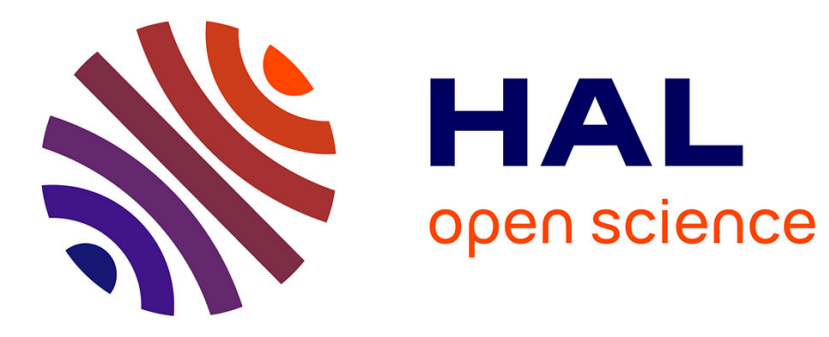

\title{
Perimeter length and form factor in two-dimensional polymer melts
}

H. Meyer, T.T. Kreer, M. Aichele, A. Cavallo, A. Johner, J. Baschnagel, J.P. Wittmer

\section{- To cite this version:}

H. Meyer, T.T. Kreer, M. Aichele, A. Cavallo, A. Johner, et al.. Perimeter length and form factor in two-dimensional polymer melts. Physical Review E: Statistical, Nonlinear, and Soft Matter Physics, 2009, 79 (5), pp.050802(R). 10.1103/PhysRevE.79.050802 . hal-00430828

\section{HAL Id: hal-00430828 \\ https://hal.science/hal-00430828}

Submitted on 22 Apr 2020

HAL is a multi-disciplinary open access archive for the deposit and dissemination of scientific research documents, whether they are published or not. The documents may come from teaching and research institutions in France or abroad, or from public or private research centers.
L'archive ouverte pluridisciplinaire HAL, est destinée au dépôt et à la diffusion de documents scientifiques de niveau recherche, publiés ou non, émanant des établissements d'enseignement et de recherche français ou étrangers, des laboratoires publics ou privés. 


\title{
Perimeter length and form factor in two-dimensional polymer melts
}

\author{
H. Meyer, T. Kreer, M. Aichele, A. Cavallo, A. Johner, J. Baschnagel, and J. P. Wittmer \\ Institut Charles Sadron, 23 rue du Loess, BP 84047, 67034 Strasbourg Cedex 2, France \\ (Received 24 June 2008; revised manuscript received 10 March 2009; published 21 May 2009)
}

\begin{abstract}
Self-avoiding polymers in two-dimensional $(d=2)$ melts are known to adopt compact configurations of typical size $R(N) \sim N^{1 / d}$, with $N$ being the chain length. Using molecular-dynamics simulations we show that the irregular shapes of these chains are characterized by a perimeter length $L(N) \sim R(N)^{d_{p}}$ of fractal dimension $d_{p}=d-\Theta_{2}=5 / 4$, with $\Theta_{2}=3 / 4$ being a well-known contact exponent. Due to the self-similar structure of the chains, compactness and perimeter fractality repeat for subchains of all arclengths $s$ down to a few monomers. The Kratky representation of the intramolecular form factor $F(q)$ reveals a strong nonmonotonous behavior with $q^{2} F(q) \sim 1 /\left(q N^{1 / d}\right)^{\Theta_{2}}$ in the intermediate regime of the wave vector $q$. Measuring the scattering of labeled subchains the form factor may allow to test our predictions in real experiments.
\end{abstract}

DOI: 10.1103/PhysRevE.79.050802

PACS number(s): 61.25.hk, 47.53.+n

\section{INTRODUCTION}

It is well known that linear polymers in two dimensions adopt compact and segregated conformations at high densities [1-6]. This is expected to apply not only on the scale of the total chain of $N$ monomers but also to subchains comprising $s$ monomers, at least as long as the segments are not too small $(1 \ll s \leq N)$. The typical size $R(s)$ of a chain segment should thus scale as $R(s) \sim s^{\nu}$ with an exponent $\nu$ $=1 / d$ set by the spatial dimension $d=2$. Compactness does obviously not imply Gaussian chain statistics [2,3] nor does segregation of chains and chain segments impose disklike shapes minimizing the average perimeter length $L(s)$ of chain segments. The boundaries of chains and of chain segments are in fact found to be highly irregular as revealed by the snapshot presented in Fig. 1. Using scaling arguments and molecular-dynamics (MD) simulations we show below that these perimeters are fractal, scaling as

$$
L(s) \sim R(s)^{d_{p}} \sim s^{1-\nu \Theta_{2}},
$$

with $d_{p}=d-\Theta_{2}=5 / 4>1$ being the fractal line dimension. Our work is based on the pioneering work by Duplantier who predicted a contact exponent $\Theta_{2}=3 / 4$ [2] characterizing the intrachain segmental size distribution. In contrast to many other possibilities to characterize numerically the compact chain conformations the perimeter length can be related to the intrachain form factor $F(q)$ making it accessible experimentally, at least in principle, by means of small-angle scattering experiments $[7,8]$.

We recall first the computational model used for this Rapid Communication and then confirm numerically the scalings of $R(s)$ and $L(s)$ suggested above. The analysis of intrachain properties such as the segmental size distribution, the bond-bond correlations, and the intrachain form factor will allow us to demonstrate Eq. (1). We conclude by discussing consequences for the dynamics of two-dimensional (2D) melts.

\section{COMPUTATIONAL ISSUES}

Our numerical results are obtained by MD simulations of monodisperse linear chains at high densities. Our coarse- grained polymer model Hamiltonian is essentially identical to the well-established Kremer-Grest (KG) bead-spring model [9-11] where the excluded volume interaction among monomers is mimicked by a purely repulsive LennardJones potential and the chain connectivity is assured by harmonic springs calibrated to the "finite extendible nonlinear elastic" (FENE) springs of the KG model. We focus in this work on melts of density $\rho=7 / 8$ at temperature $k_{B} T=1$ with chain lengths ranging up to $N=2048$ using a periodic simulation box of linear length 335.2 containing 98304 monomers [12].

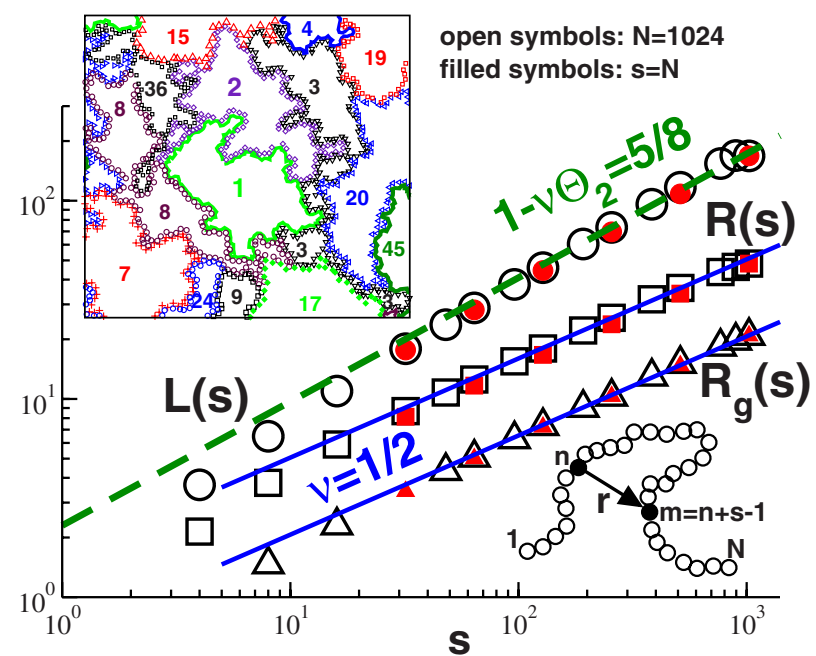

FIG. 1. (Color online) A snapshot of a melt configuration with chain length $N=1024$ and monomer density $\rho=7 / 8$ can be seen in the left panel. Only the perimeter monomers interacting with other chains are indicated. The numbers refer to an arbitrary chain index used for computational purposes. The chains are compact, i.e., they fill space densely, but compactness does not imply a disklike shape (see, e.g., chains 3 and 8). The main figure presents the end-to-end distance $R(s)=\left\langle\mathbf{r}^{2}\right\rangle^{1 / 2}$, the radius of gyration $R_{g}(s)$, and the mean perimeter length $L(s)$ of segments containing $s=m-n+1$ monomers as indicated by the sketch on the right. The full symbols refer to overall chain properties $(s=N)$. The solid lines confirm the exponent $\nu=1 / 2$ for the segment size, the dashed line confirms the scaling of $L(s)$ suggested by Eq. (1). 


\section{MEAN SEGMENT SIZE AND PERIMETER LENGTH}

The main part of Fig. 1 presents the typical size and perimeter length of a chain segment between the monomers $n$ and $m=n+s-1$ as indicated by the sketch. Following $[13,14]$ we average over all pairs $(n, m)$ possible in a chain of length $N$. Averaging only over segments at the curvilinear chain center slightly reduces chain end effects, however the difference is negligible for the larger chains, $N>256$, we will focus on. Open symbols refer to segments of length $s \leq N$ of chains of length $N=1024$, full symbols to total chain properties $(s=N)$. The segment size may be characterized by either the second moment $R^{2}(s)=\left\langle\mathbf{r}^{2}\right\rangle$ of the end-to-end vector $\mathbf{r}$ of the segment (squares) or by its radius of gyration $R_{g}^{2}(s)$ (triangles) [15]. In agreement with various numerical studies [4-6] the presented data confirms that the chains are compact, i.e., $\nu=1 / 2$ (solid lines), on all scales $s$ [16]. A perimeter monomer of a chain segment is defined as a monomer being within a distance of 1.2 to a monomer not belonging to the same chain segment [17]. The mean number $L(s)$ of these perimeter monomers increases with a power-law exponent $1-\nu \Theta_{2}=5 / 8$ (dashed line) which is in perfect agreement with Eq. (1), and this holds again on all scales for arbitrary segment lengths provided that the segment is sufficiently large $(s>50)$. We demonstrate in the following where the suggested scaling stems from.

\section{SEGMENT SIZE DISTRIBUTIONS AND CONTACT EXPONENTS}

Obviously, the mere fact that the exponent $\nu$ is the same in two and three dimensions does not imply that 2D melts are Gaussian [2]. This can be directly seen from the different probability distributions of chain segment vectors $\mathbf{r}=\mathbf{r}_{m}-\mathbf{r}_{n}$ presented in Fig. 2. To simplify the plot we focus on the two longest chains simulated, $N=1024$ and $N=2048$. $G_{0}(r, N)$ characterizes the distribution of the total chain end-to-end vector $(n=1, m=N), G_{1}(r, N)$ characterizes the distance between a chain end and the monomer in the middle of the chain $(n=1, m=N / 2)$, and $G_{2}(r, N)$ characterizes the distribution of an inner segment vector between the monomers $n=N / 4$ and $m=3 N / 4$. In addition, we indicate the segmental size distribution $G(r, s)$ averaging over all pairs $(n, m)$ which has been used recently to characterize deviations from ideal chain behavior in three-dimensional (3D) melts [14]. All data for different $N$ and $s$ collapse on three distinct master curves if the axes are made dimensionless using the second moment $R_{i}^{2}$ of the respective distribution as indicated in the figure. The only relevant length scale is thus the typical size of the segment itself. The distributions are not monotonous and are thus qualitatively different from the Gaussian (thin lines) expected for random walks. In agreement with Duplantier [2] we find

$$
R_{i}^{d} G_{i}(r, N)=x^{\Theta} f_{i}(x),
$$

with $x=r / R_{i}$ being the scaling variable and the contact exponents $\Theta_{0}=3 / 8, \Theta_{1}=1 / 2$, and $\Theta_{2}=3 / 4$ (dashed lines) describing the small- $x$ limit where the universal functions $f_{i}(x)$ become constant. Especially the largest of these exponents,

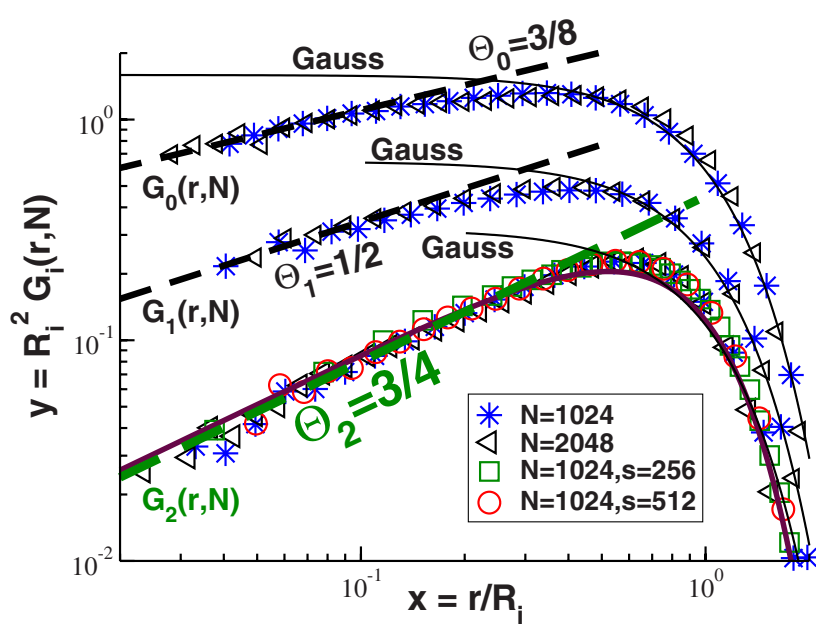

FIG. 2. (Color online) Scaling plot of various distributions of the end-to-end vector $\mathbf{r}=\mathbf{r}_{m}-\mathbf{r}_{n}$ of chain segments of chains of length $N=1024$ (stars) and $N=2048$ (triangle): $G_{0}(r, N)$ for $n=1$ and $m=N, G_{1}(r, N)$ for $n=1$ and $m=N / 2, G_{2}(r, N)$ for $n=N / 4$ and $m=3 N / 4 . G_{0}(r, N)$ and $G_{1}(r, N)$ are shifted vertically for clarity. The segmental size distribution $G(r, s)$ averaging over all pairs $(n, m)$ given for $N=1024$ with $s=256$ (squares) and $s=512$ (spheres) scales as $G_{2}(r, N)$. The thin lines indicate the Gaussian distribution $y=\exp \left(-x^{2}\right) / \pi$ expected for ideal chains in two dimensions. The power laws $y \approx x^{\Theta_{i}}$ (dashed lines) observed for $x \ll 1$ have been predicted by Duplantier [2]. The Redner-des Cloizeaux formula for $G_{2}(r, N)$ is indicated by a solid line.

$\Theta_{2}$, is clearly visible. The contact probability for two monomers of a chain in a $2 \mathrm{D}$ melt is thus strongly suppressed compared to ideal chain statistics $\left(\Theta_{0}=\Theta_{1}=\Theta_{2}=0\right)$. As can be seen, the rescaled distributions $G(r, s)$ and $G_{2}(r, N)$ become identical for intermediate segment length, $1 \ll s \ll N$. [Obviously, $G(r, s) \approx G_{0}(r, N)$ for very large segments $s \rightarrow N$.] It is for this reason that the exponent $\Theta_{2}$ is the most important one for asymptotically long chains where chain end effects can be neglected. The rescaled distributions show exponential cutoffs for large distances. The Redner-des Cloizeaux formula [18] is a useful interpolation formula which supposes that $f_{i}(x)=C_{i} \exp \left(-K_{i} x^{2}\right)$ with constants $C_{i}$ and $K_{i}=1+\Theta_{i} / 2$ imposed by the normalization and the second moment of the distributions [19]. This formula is by no means rigorous but yields reasonable parameter free fits as shown for $f_{2}(x)$.

\section{BOND-BOND CORRELATIONS}

The bond-bond correlation function $P_{1}(s)=\left\langle\mathbf{e}_{n} \cdot \mathbf{e}_{m}\right\rangle\left(\mathbf{e}_{i}\right.$ denoting the normalized bond vector connecting the monomers $i$ and $i+1$ ) has been shown to be of particular interest for characterizing the deviations from random-walk statistics in $3 \mathrm{D}$ polymer melts $[13,14]$. The reason for this is that $P_{1}(s)$ is proportional to the second derivative of the segment size $R(s)^{2}$ with respect to segment length $s$ so that small deviations from the asymptotic exponent $2 \nu=1$ are emphasized [14]. As can be seen from the inset in Fig. 3 deviations of this kind are small for large $s$ and may be neglected for the present Rapid Communication. The main effect visible is an 


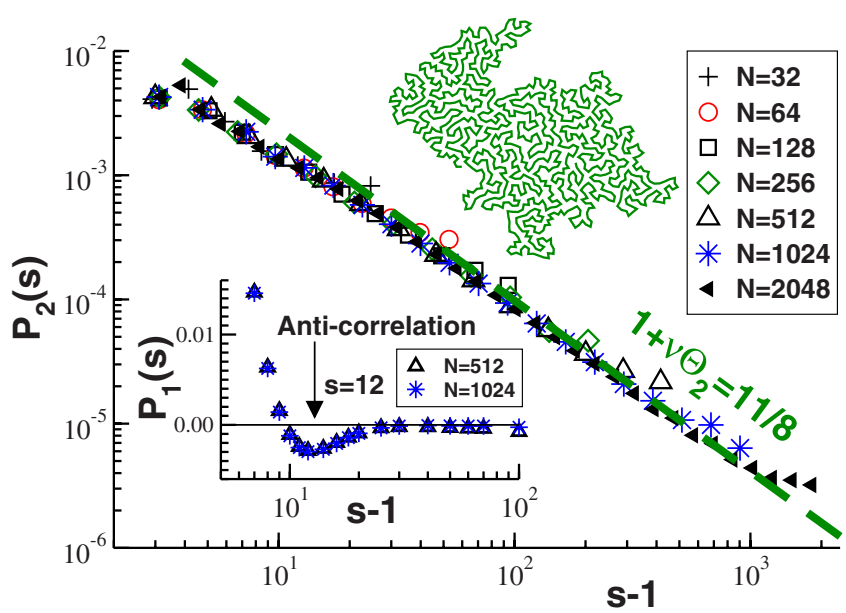

FIG. 3. (Color online) Bond-bond correlation functions $P_{1}(s)=\left\langle\mathbf{e}_{n} \cdot \mathbf{e}_{m}\right\rangle$ and $P_{2}(s)=\left\langle\left(\mathbf{e}_{n} \cdot \mathbf{e}_{m}\right)^{2}\right\rangle-1 / 2$ vs curvilinear distance $s-1$ between normalized bond vectors $\mathbf{e}_{n}$ and $\mathbf{e}_{m}$. The first Legendre polynomial $P_{1}(s)$ (inset) shows an anticorrelation at $s \approx 12$. The second Legendre polynomial $P_{2}(s)$ decays over 2 orders in magnitude as a power law (dashed line) with an exponent $1+\nu \Theta_{2}=11 / 8$ in agreement with the return probability calculated from Eq. (2).

anticorrelation at $s \approx 12$ due to the backfolding of the chain contour which can be directly seen from the snapshot. Conceptually more important is the fact that the second Legendre polynomial $P_{2}(s)=\left\langle\left(\mathbf{e}_{n} \cdot \mathbf{e}_{m}\right)^{2}\right\rangle-1 / 2$ reveals a clear power-law behavior over 2 orders of magnitude in $s$ (dashed line). The power law is due to the alignment of two bonds if they are sufficiently close, i.e., the exponent measures the return probability after $s$ steps. It follows from Eq. (2) that for 1 $\ll s \ll N$ this is given by $\lim _{r \rightarrow 0} G(r, s) \sim 1 / s^{1+\nu \Theta_{2}}=s^{-11 / 8}$. The agreement of the data with this exponent is excellent and provides an independent confirmation of $\Theta_{2}=3 / 4$.

\section{INTRACHAIN FORM FACTOR}

Neither segmental size distributions nor bond-bond correlation functions are readily accessible experimentally. It is thus important that $\Theta_{2}$ should be measurable - at least in principle-from an analysis of the intrachain form factor $F(q)=\frac{1}{N} \sum_{n, m=1}^{N}\left\langle\exp \left[i \mathbf{q} \cdot\left(\mathbf{r}_{n}-\mathbf{r}_{m}\right)\right]\right\rangle$. The reason for this is that the form factor can be expressed by the Fourier transform $G(q, s)=\left\langle\exp \left[i \mathbf{q} \cdot\left(\mathbf{r}_{n}-\mathbf{r}_{m}\right)\right]\right\rangle_{n m}$ of the segmental size distribution $G(r, s)$, with $\mathbf{q}$ being the wave vector: $F(q)$ $=\frac{2}{N} \int_{0}^{N} d s(N-s) G(q, s)$. Assuming $G(r, s) \approx G_{2}(r, N)$ and using the Redner-des Cloizeaux approximation in Eq. (2) this yields a lengthy analytic formula (not given) which is represented by the solid line in Fig. 4 [20]. For wave vectors corresponding to the power-law regime of Eq. (2) this reduces to the simple power law,

$$
F(q) / N \approx 1.98 /\left[q R_{g}(N)\right]^{d+\Theta_{2}},
$$

indicated by the dashed line. Note that the above scaling is a direct consequence of $G(r, s) \sim r^{\Theta_{2}}$ and does not rely on the Redner-des Cloizeaux approximation. We rescale the wave vector with the measured radius of gyration $R_{g}(N)$ (presented in Fig. 1) to collapse all data in the Guinier regime for small

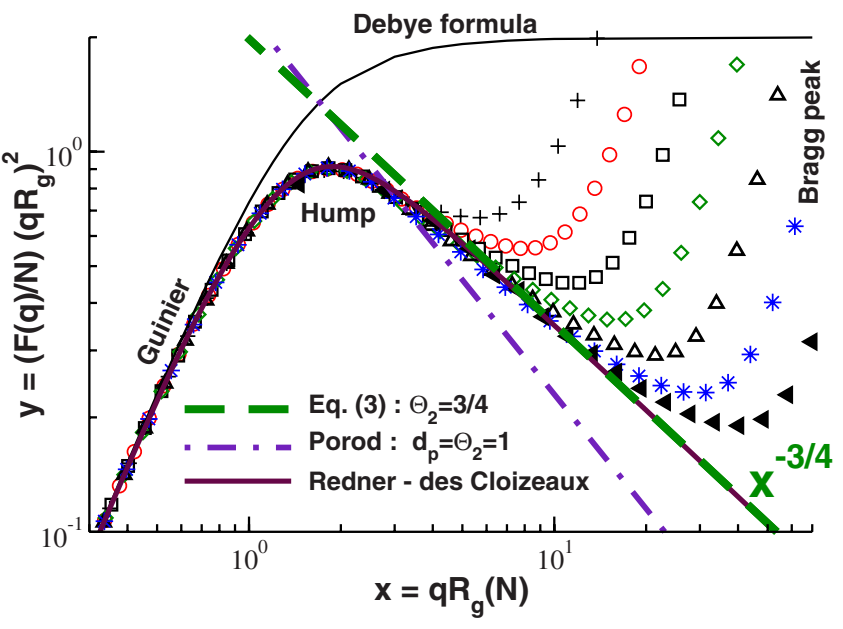

FIG. 4. (Color online) Kratky representation of the intramolecular form factor $F(q)$ as a function of $x=q R_{g}(N)$ for different chain lengths $N$ using the same symbols as in Fig. 3. The Debye formula (thin line) corresponds to a chain length independent plateau for $x \gg 1$. By contrast to this, a strong nonmonotonous behavior is revealed by our data which approaches with increasing $N$ a power-law exponent $-\Theta_{2}=-3 / 4$ (dashed line) corresponding to a compact object of fractal line dimension $d_{p}=d-\Theta_{2}=5 / 4$. Also included is the Porod scattering expected for a compact $2 \mathrm{D}$ object with smooth perimeter (dash-dotted line) and the Fourier transform of the Redner-des Cloizeaux approximation (solid line). The increase in the scattering for large $q$ (Bragg peak) is due to the packing of the beads on local scale. Only in this limit does $F(q)$ become chain length independent.

$x=q R_{g}(N) \ll 1$ and use a Kratky representation for the vertical axis $y=[F(q) / N] x^{2}$. While $y$ becomes constant and independent of chain length for $x \gg 1$ for Gaussian chains (as shown by the Debye formula indicated) [1], we observe over a decade in $x$ a striking nonmonotonous behavior. Our data suggests that Eq. (3) is approached systematically with increasing chain length - the central numerical result presented in this Rapid Communication.

\section{IDENTIFICATION OF $\Theta_{2}$ AND THE FRACTAL LINE DIMENSION}

The preceding discussion focused exclusively on intrachain properties. Since 2D chains are compact (Fig. 1) only monomers on the chain perimeter interacting with monomers from other chains can contribute to the scattering. Quite generally, the scattering intensity $N F(q)$ of compact objects becomes proportional to the mean "surface" $L(N) \sim R(N)^{d_{p}}$ for $q R(N) \gg 1$ which implies the generalized Porod law $[7,21,22]$

$$
N F(q) \approx N^{2} /[q R(N)]^{2 d-d_{p}} .
$$

For a $2 \mathrm{D}$ object with smooth perimeter $\left(d_{p}=1\right)$ this corresponds to the classical Porod scattering $F(q) \sim 1 / q^{3}$ represented by the dash-dotted line in Fig. 4. Comparing Eq. (4) with Eq. (3) shows that the 2D melts are characterized by a fractal line dimension $d_{p}=d-\Theta_{2}$ and demonstrates finally the scaling of the perimeter length $L(N)$ postulated in Sec. I and verified numerically in Fig. 1. By labeling only the mono- 
mers of subchains [which corresponds to scattering amplitude $\left.s F(q) \sim L(s) \sim R(s)^{d} p\right]$ the above argument is readily generalized to the perimeter length $L(s)$ of arbitrary segment length $s \leq N[23]$.

\section{SUMMARY}

Investigating various static properties of linear polymer melts in two dimensions, we have demonstrated that the compact chains and chain segments $(\nu=1 / 2)$ are characterized by a fractal perimeter $L(s)$ of line dimension $d_{p}=d$ $-\Theta_{2}=5 / 4$. As may be seen from Fig. 4, computationally very demanding systems with chain length $N>10^{3}$ are required and have thus been simulated to put the suggested scaling behavior to the test. Our results may be verified experimentally from the scaling of the intrachain form factor $F(q)$ whose Kratky representation is predicted to reveal a strong nonmonotonous behavior. This should also hold in semidilute solutions provided that the chains are long enough [12]. Interestingly, the fractality of the perimeter precludes a finite line tension. Thus, shape fluctuations of the segments are not suppressed exponentially [4] but may occur in an "amoebalike" fashion by advancing and retracting "lobes." According to a recent suggestion [3] the relaxation time $\tau(s)$ of a chain segment may, hence, scale as $\tau(s) \sim L(s)^{3} \sim s^{15 / 8}$ rather than as $s^{2}$ as in the standard Rouse model [15]. Clearly, as Gaussian chain statistics is inappropriate to describe conformational properties of $2 \mathrm{D}$ melts, there is no reason why a model based on this statistics should allow to describe, e.g., the perimeter length fluctuations. Since the latter property is in principle accessible experimentally from the dynamical intrachain structure factor $F(q, t)[7,15]$ this is an important issue we are currently investigating.

\section{ACKNOWLEDGMENTS}

We thank the ULP, the IUF, the Deutsche Forschungsgemeinschaft (Grant No. KR 2854/1-1), and the ESFSTIPOMAT program for financial support. A generous grant of computer time by the IDRIS (Orsay) is also gratefully acknowledged. We are indebted to A. N. Semenov for helpful discussions.
[1] P. G. de Gennes, Scaling Concepts in Polymer Physics (Cornell University Press, Ithaca, New York, 1979).

[2] B. Duplantier, J. Stat. Phys. 54, 581 (1989).

[3] A. Semenov and A. Johner, Eur. Phys. J. E 12, 469 (2003).

[4] I. Carmesin and K. Kremer, J. Phys. (France) 51, 915 (1990).

[5] A. Cavallo, M. Müller, and K. Binder, J. Phys. Chem. B 109, 6544 (2005)

[6] A. Yethiraj, Macromolecules 36, 5854 (2003).

[7] J. Higgins and H. Benoît, Polymers and Neutron Scattering (Oxford University Press, Oxford, 1996).

[8] B. Maier and J. O. Radler, Phys. Rev. Lett. 82, 1911 (1999).

[9] G. S. Grest and K. Kremer, Phys. Rev. A 33, 3628 (1986).

[10] K. Kremer and G. Grest, J. Chem. Phys. 92, 5057 (1990).

[11] S. J. Plimpton, J. Comput. Phys. 117, 1 (1995).

[12] The presented data was obtained on IBM power 6 with the LAMMPS version 21 May 2008 [11] and is part of a larger study where we have systematically varied density, system size, and friction coefficient of the Langevin thermostat to confirm the robustness of theory and simulation with respect to these parameters. Taken apart the longest chains with $N=2048$ all chains have diffused over at least 10 times their radius of gyration providing thus high-precision statistics.

[13] J. P. Wittmer, H. Meyer, J. Baschnagel, A. Johner, S. P. Obukhov, L. Mattioni, M. Müller, and A. N. Semenov, Phys. Rev. Lett. 93, 147801 (2004).

[14] J. P. Wittmer, P. Beckrich, H. Meyer, A. Cavallo, A. Johner, and J. Baschnagel, Phys. Rev. E 76, 011803 (2007).

[15] M. Doi and S. F. Edwards, The Theory of Polymer Dynamics (Clarendon Press, Oxford, 1986).

[16] The log-log representation chosen in Fig. 1 masks deliberately corrections to the leading power law $\nu=1 / 2$ due to finite segment and chain lengths which exist in two dimensions as they do in three dimensions [14]. These are revealed, e.g., by a logarithmic-linear plot of $R^{2}(s) / s$ vs $s$ showing a nonmonotonous behavior with a sharp decay for $s \rightarrow N$ where the $\Theta_{0}$ exponent becomes relevant. It is ultimately due to this decay that the ratio $R^{2}(N) / R_{g}^{2}(N)$ approaches a value 5.3 rather than 6.

[17] We have checked that the scaling of $L(s)$ does not depend on the distance used for defining a perimeter monomer.

[18] J. des Cloizeaux and G. Jannink, Polymers in Solution: Their Modeling and Structure (Clarendon Press, Oxford, 1990).

[19] R. Everaers, I. Graham, and M. Zuckermann, J. Phys. A 28, 1271 (1995).

[20] Since we use only the $\Theta_{2}$ exponent and in addition to this the Redner-des Cloizeaux formula there are two levels of approximation. Our final result is rephrased consistently in terms of $x=q R_{g}(N)$ and, thus, by construction our formula yields correctly both the Guiner regime for $x \ll 1$ and the generalized Porod limit for $x \gg 1$, but not necessarily the hump region at $x \approx 2$. In practice, our approximation is fine for all $x$ as shown by Fig. 4 .

[21] P. Z. Wong and A. J. Bray, Phys. Rev. Lett. 60, 1344 (1988).

[22] H. D. Bale and P. W. Schmidt, Phys. Rev. Lett. 53, 596 (1984).

[23] An alternative derivation of Eq. (1) is obtained from a scaling argument using the return probability $1 / s^{1+\nu \Theta_{2}}$ measured in Fig. 3. The key point is that a monomer in a long segment cannot "distinguish" if the contact is realized through the backfolding of its own segment or by another segment [2,3]. Since the probability of such a binary contact, $L(s) / s$, must be proportional to the return probability times the number $s$ of monomers in the second segment this yields Eq. (1). 